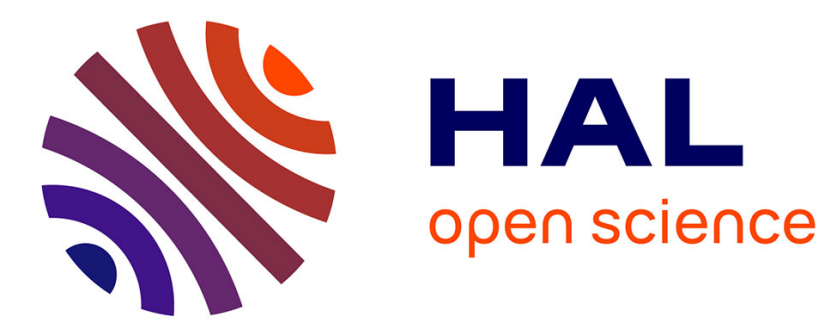

\title{
Les Film Festival Studies, éléments pour une épistémologie d'un nouveau champ de recherche
}

Christel Taillibert

\section{To cite this version:}

Christel Taillibert. Les Film Festival Studies, éléments pour une épistémologie d'un nouveau champ de recherche. Diogène: Revue internationale des sciences humaines, 2017. halshs-02938647

\section{HAL Id: halshs-02938647 https://shs.hal.science/halshs-02938647}

Submitted on 15 Sep 2020

HAL is a multi-disciplinary open access archive for the deposit and dissemination of scientific research documents, whether they are published or not. The documents may come from teaching and research institutions in France or abroad, or from public or private research centers.
L'archive ouverte pluridisciplinaire HAL, est destinée au dépôt et à la diffusion de documents scientifiques de niveau recherche, publiés ou non, émanant des établissements d'enseignement et de recherche français ou étrangers, des laboratoires publics ou privés. 


\section{LES FILM FESTIVAL STUDIES, ELEMENTS POUR UNE EPISTEMOLOGIE D’UN NOUVEAU CHAMP DE RECHERCHE}

par

CHRISTEL TAILLIBERT

Depuis le début des années 2010 fleurissent les publications, les rencontres, les formations qui se placent sous le signe des Film Festival Studies. Depuis longtemps, pourtant, les festivals de films avaient rencontré l'intérêt de chercheurs, issus de diverses disciplines. Une dynamique nouvelle serait-elle donc à l'œuvre depuis la dernière décennie autour de cette volonté explicite de se raccrocher à la perspective très anglosaxonne des Studies? Ce texte se propose d'ouvrir quelques pistes de réflexion relatives à la spécificité des études portant sur cet objet singulier que constituent les festivals de cinéma et audiovisuel, dans la perspective de poser des jalons propres à l'épistémologie d'un champ de recherche encore à construire. Il se déclinera en deux temps. Nous introduirons notre réflexion en tentant de comprendre quelles sont les spécificités des études propres aux festivals de cinéma et audiovisuels au sein du champ plus large des Festivals Studies. Dans un second temps, sur la base des caractéristiques mises en évidence, nous proposerons quelques points de repères propres à l'élaboration d'une épistémologie des Film Festival Studies.

Les Films Festival Studies au prisme des Festival Studies :

singularité d'un champ d'étude

Poser l'existence d'un champ spécifique d'étude pour les Film Festivals Studies suppose que ce dernier puisse affirmer son autonomie par rapport à d'autres champs de recherche, plus vastes, en particulier par rapport aux Festivals Studies. Ce dernier se présente comme un champ structuré, notamment dans le monde anglo-saxon. Le bilan dressé par Donald Getz, dès 2010, témoigne de sa vitalité, mais aussi de sa capacité à 
penser ses objectifs et les moyens de son action, quand bien même pensée ici dans le cadre du management touristique et événementiel. En quoi les festivals de films devraient-ils, dans ce contexte, constituer un champ de recherche autonome ? Pourquoi les outils mis en œuvre devraient-ils différer de ceux qui ont été construits pour comprendre le monde festivalier dans son ensemble ? Nous tenterons de montrer en quoi la particularité de ce champ d'études impose la construction d'un cadre épistémique singulier.

Un cadre économique spécifique, entre économie de soutien et puissants enjeux industriels

Le premier point à souligner tient logiquement aux enjeux économiques qui accompagnent le développement de l'industrie cinématographique et audiovisuelle, et qui conditionnent les festivals s'y rattachant. C'est une banalité de rappeler le fait qu'au sein des industries culturelles, l'industrie cinématographique et audiovisuelle génère des flux financiers phénoménaux. Les festivals qui relèvent du vaste secteur des moving images ne peuvent donc pas être pensés en dehors de leur fonction de régulation au sein de ce marché global, et en particulier au prisme de leur rôle de vitrine culturelle conférant par nature une valeur symbolique aux films qu'ils accueillent - et ce, de façon bien plus marquée que pour des festivals travaillant au cœur d'autres types d'industries culturelles. Cette affirmation tient au caractère fréquemment compétitif de ces manifestations : les prix qu'ils délivrent traduisent, dans le processus de reconnaissance artistique qui fonde leur raison d'être, une volonté explicite d'infléchir le marché, d'influer sur les décisions de consommation des spectateurs, en conférant une plus-value symbolique à des films choisis. Bien entendu, différents types de distinction jouent un rôle similaire dans d'autres champs propres aux industries culturelles (tels les prix littéraires, aux conséquences économiques indéniables, mais aussi, dans une moindre mesure, en termes d'impact les diverses récompenses qui ponctuent le calendrier théâtral ou musical), mais ce ne sont pas les festivals qui sont habilités à les délivrer, contrairement au secteur cinématographique où ils participent pleinement - sans en avoir l'exclusivité - à cette économie de la récompense. Ce rôle est joué en premier lieu par les grands festivals internationaux, en particulier pour les films au potentiel international avéré, mais aussi 
par des festivals plus modestes, qui contribuent à conférer une reconnaissance symbolique importante à des films d'auteur au destin plus incertain : ceux-ci peuvent ainsi envisager une carrière honorable dans les réseaux Art \& Essai au moment de leur sortie en salle - notamment en France où ce secteur bénéficie de l'existence de catégories de spectateurs cinéphiles porteuses pour ces films.

La spécificité nationale dont nous venons de faire état nous amène à développer un second point propre à la spécificité économique du champ festivalier cinématographique : le modèle français qui encadre l'industrie cinématographique et audiovisuelle, porté par une économie de soutien structurée sur la base des marqueurs forts de la politique culturelle nationale qui donne la part belle à l'auteurisme et à l'innovation, explique aussi - la vitalité hors norme du secteur festivalier français dans ce domaine particulier (Taillibert 2009). Cette politique est marquée par la volonté d'opposer une résistance culturelle et économique à l'hégémonie hollywoodienne. Elle a trouvé, dans l'aprèsguerre, sa traduction dans le concept $d$ '« exception culturelle », toujours très vivace dans le discours des responsables des festivals de films français, qui n'hésitent pas à se placer sous une bannière militante, mettant la question de la résistance culturelle au premier plan de leur action.

Cette économie de soutien promue par l'État français dans le champ cinématographique tend par ailleurs à favoriser l'innovation, l'expression par le cinéma d'une sensibilité artistique, orientations qui trouvent leur traduction dans une politique ambitieuse de soutien à la production, au niveau des soutiens sélectifs du CNC, mais aussi des politiques de production impulsées par les Régions qui sont aujourd'hui extrêmement prolifiques. Or, les films nés de cette dynamique ne trouvent pas tous, loin s'en faut, une place sur le marché de la distribution commerciale classique - salles, télévision, vidéo - et même les plus privilégiées ne bénéficient souvent que d'une sortie salle technique. Pour tous ces films, que l'on qualifiera de « films sans distributeur », les festivals jouent un rôle essentiel puisqu'ils permettent de rencontrer un public, de faire jouer le bouche-à-oreille, de créer une réputation aux auteurs, voire de permettre des retours sur investissement. On parle beaucoup du rôle de marché informel joué de fait par les festivals, mais moins de ce réseau de distribution parallèle que constitue ce maillage de festivals de films, non seulement en France mais aussi en Europe puisque nombreux 
sont les échanges de films pratiqués entre les différents partenaires. On peut donc avancer l'hypothèse que la politique de soutien à la production de l'État français, au niveau central comme au niveau régional, existe uniquement grâce aux festivals de films situation qui ne trouve pas de pendant dans les autres secteurs culturels. Le fait que l'on désigne certaines catégories de production comme étant des « films de festivals» tend à corroborer cette affirmation.

\section{L'historicité des modèles de médiation cinéphilique}

Le second point que nous souhaiterions soulever concerne le rapport très étroit entretenu par les festivals de films avec le concept de cinéphilie (De Baecque 2003 ; De Valck, Hagener 2005, etc.). Ce rapport particulier au cinéma, éminemment français dans sa désignation comme dans sa nature, n'existe pas en tant que tel pour les autres arts ou expressions culturelles. Il implique une relation passionnelle, mais qui va au-delà des pratiques de fans, modelée sur une appréhension à la foi quantitative du rapport au cinéma (un cinéphile voit beaucoup de films), mais aussi qualitative puisque les films sont jaugés à l'aune de critères de jugements, d'essence artistiques, à la fois personnels et répondant à une norme partagée ; ce mode d'appréciation de l'art cinématographique est intimement lié à des questions d'éducation, laquelle permet de travailler la compétence du spectateur, et peut être considéré comme éminemment politique puisqu'il s'inscrit dans une vision dichotomique du cinéma, partagé entre une production industrielle de divertissement d'une part et une production auteuriste et culturelle d'autre part, qui induit une visée dans l'infléchissement des politiques industrielles; le cinéphile est ainsi tout entier défini par le rapport singulier qu'il entretient avec cet art, et inscrit sa pratique dans une histoire - politique, économique et culturelle - des pratiques culturelles cinématographiques.

Si la question de la cinéphilie nous semble essentielle pour comprendre la spécificité des festivals de films dans le champ festivalier, c'est parce que celle-ci s'est construite au gré de l'expérimentation d'un certain nombre de dispositifs de médiation - les ciné-clubs, les salles art \& essai, les cinémathèques - dont les festivals sont, dans leur très grande majorité, directement les héritiers. Ainsi, étudier les festivals de films aujourd'hui ne peut 
s'entendre en dehors de la compréhension de cet écosystème cinéphile global, tout entier tourné vers le besoin d'éducation du spectateur. D'où la redondance dans les modalités d'action, entre l'étayage informationnel lié à l'idée d'un savoir à constituer, l'organisation de débats destinés à travailler l'émancipation intellectuelle des spectateurs pris individuellement, mais aussi «le devenir d'un public participant» pour reprendre l'expression d'Emmanuel Ethis (2007: 15). Impossible, donc, de travailler sur les festivals de films sans comprendre cet horizon d'attente qui traverse les pratiques cinéphiles, et que l'on ne retrouvera pas sous cette forme dans les autres festivals culturels - qui, même traversés d'une dimension éducative, limitent davantage le cadre de la médiation à la présentation des œuvres, prenant moins directement en charge la question de l'accompagnement du spectateur comme peuvent le faire les festivals de films.

\section{L’image animée : un média du quotidien et un réservoir thématique inépuisable}

Considérer la spécificité des festivals de films, c'est aussi prendre conscience de la capacité du média audiovisuel à aborder absolument n'importe quel sujet - ce qui encore une fois ne se vérifie pas pour les autres productions culturelles représentées en festivals. Ce point est important, car il explique pourquoi des acteurs de nature très variée sont amenés à s'investir dans cette forme d'événementiel. Si le film est le plus souvent envisagé en festival au prisme de son statut d'œuvre d'art, il constitue aussi un média idéal pour communiquer sur des sujets de toute nature. D'où la présence dans le secteur festivalier d'acteurs qui n'ont, au départ, aucun intérêt particulier pour ce média, et qui ne l'envisagent que comme vecteur de leur action de communication - c'est le cas, par exemple, des associations militantes qui créent des festivals de films pour relayer leur parole (on peut évoquer les groupements LGBT, les associations en faveur du droit des personnes en situation de handicap, etc.), des centres et instituts culturels qui organisent des festivals pour diffuser la culture et la langue française, des associations qui entendent mettre en lumière leur activité par une opération événementielle (un exemple parmi tant d'autres, le Festival du Film d'Archéologie d'Amiens organisé par le C.I.R.A.S., le Centre Interdisciplinaire de Recherches Archéologiques de la Somme), etc. 
On observe donc une réelle diversité d'approche dans les pratiques festivalières, spécifiquement dans le domaine du film, qui induit directement une hétérogénéité des publics de ces manifestations : à côté des cinéphiles, on trouvera des publics très variés en fonction de la raison d'être de telle ou telle manifestation, élargissant ainsi l'impact de ce secteur d'un point de vue sociologique.

Cette capacité des festivals de films à toucher tous les publics s'explique aussi par le fait qu'aucune barrière psychologique ou culturelle n'entrave la participation à une projection d'images animées : ce média, qu'il soit perçu comme source d'informations, de divertissement ou de culture, appartient au quotidien de tous les publics, quelle que soit sa classe sociale d'appartenance, son niveau d'étude, son âge ou son origine. Ces dispositifs de médiation que constituent les festivals de films voient ainsi leur travail facilité dans la mesure où l'image animée, contrairement à d'autres expressions artistiques et culturelles, n'est pas sociologiquement porteuse de distinction sociale. C'est un point très important pour comprendre et étudier la façon dont se sont développées ces manifestations, dans toute la diversité de leurs propositions, par exemple dans le champ de l'éducation populaire. Les associations issues de ce projet de société ont en effet largement envisagé l'image animée comme un outil d'action privilégié, justement parce qu'elle était susceptible d'attirer des publics populaires autour d'un projet culturel, citoyen, émancipateur, sans susciter de réticences - et comme on l'a vu susceptible d'aborder des sujets de toutes sortes, d'interroger le monde et ses évolutions.

\section{$\underline{\text { L’image animée au cœur des pratiques contemporaines }}$}

Le dernier point concerne la question des pratiques amateur, et de leur véritable explosion au cours des dernières décennies. Si la pratique du "cinéma amateur » est ancienne, historiquement liée aux formats réduits cinématographiques, puis à la vidéo, deux phénomènes expliquent l'actuel développement intensif de ces pratiques : tout d'abord la démocratisation d'outils de production de qualité (au niveau des appareils de

prise de vues, des logiciels de montage et de post-production, etc.) mais aussi et surtout le développement d'Internet, qui offre des espaces infinis d'exposition et de communication des contenus amateur, dans une logique de désintermédiation quasi-totale. Si ce «sacre de l'amateur » que célébrait Patrice Flichy (2010) nous intéresse ici, c'est qu'il est à 
l'origine d'une augmentation impressionnante du nombre de productions filmiques, laquelle va aussi de pair avec une augmentation qualitative desdites productions, les échanges en ligne ayant aussi permis un meilleur partage de l'expertise.

À côté du tout-venant, on trouve beaucoup de productions que l'on peut qualifier de semi-professionnelles qui n'ont, par nature, pas vocation à intégrer le circuit commercial, mais qui vont rencontrer l'intérêt des organisateurs de festivals de films. Rappelons que la création occupe la part belle dans les outils d'éducation à l'image, et que cette recrudescence des pratiques ne peut que satisfaire les objectifs poursuivis par ces acteurs. C'est ainsi que l'on observe une multiplication de festivals de films amateurs qui vont constituer des vitrines pour les plus talentueuses de ces productions, vitrines qui, contrairement aux plateformes de partage sur Internet, vont proposer une véritable éditorialisation des contenus et une reconnaissance du geste artistique et créatif des auteurs. Les festivals de court métrage, quand bien même ils ne sont pas estampillés « amateur », se nourrissent de ce type de productions.

Encore une fois, ce phénomène ne trouve absolument pas son pendant dans les autres types de festivals. S'il existe quelques festivals de théâtre amateur, accueillant les troupes locales, ils n'offrent guère davantage qu'une extension du concept de spectacle de fin d'année, et si les festivals de musique plongent leurs racines dans la tradition orphéonique (Gumplowicz 1987), on n'en trouve plus l'expression aujourd'hui dans les festivals contemporains - seule la Fête de la musique pouvant offrir l'exemple d'un événementiel musical largement porté par les non-professionnels.

Les études portant sur les festivals de films ne pourront donc faire abstraction de ce statut particulier de l'amateurisme dans le domaine cinématographique au sens large, intimement corrélé à d'autres phénomènes que nous avons soulevés précédemment, à savoir le travail d'éducation à l'image qui accompagne la valorisation de ces pratiques « amateur » en festival, les ponts qu'elles permettent vers une cinéphilie plus construite et plus revendiquée, mais aussi l'outil qu'elles constituent au service de projets d'éducation populaire. Au sein des presque 700 festivals annuels que compte d'actualité culturelle cinématographique française, l'art et l'esthétique en tant que valeurs distinctives, caractéristiques d'une perception très cinéphilique de la fonction festivalière, côtoient ainsi d'autres valeurs - sociales, éducatives - qui constituent la charpente symbolique 
d'autres typologies de festivals tout autant significatives de la réalité du secteur. Au terme de cette première approche, et même si de nombreux autres points mériteraient d'être développés, nous poserons le principe selon lequel il est tout à fait légitime d'isoler le champ des Film Festival Studies au sein de celui, plus vaste, des Festivals Studies : audelà de la proximité évidente qu'entretiennent les festivals de films avec les autres types de festivals, dans leur relation à la proposition événementielle, dans leur fonction dans l'économie et la politique locale, etc., le cadre très particulier - économique, historique, culturel et symbolique - dans lequel évolue ces manifestations justifie sa construction comme champs de recherche part entière. Mais ces Film Festival Studies dont nous venons de justifier l'épanouissement existent-elles? Qu'en est-il de ce champ de recherche aujourd'hui?

Approche épistémologique des Film Festivals Studies

Le concept de Film Festival Studies auquel ont aujourd'hui recours un certain nombre de chercheurs pour caractériser leur terrain d'investigation trouve ses origines dans une volonté de structurer un champ de recherche dont les manifestations les plus évidentes se situent au tournant des années 2010.

Le premier marqueur fort de cette volonté est lié à la création en 2008, par Marijke de Valck et Skadi Loist, d'un réseau destiné à regrouper les chercheurs travaillant sur le même objet : le Film Festival Research Network. En recensant les travaux d'ores et déjà publiés sur le sujet, en communiquant sur les travaux en cours, ce réseau a permis rapidement de conférer une identité, un cadre, à cet objet de recherche spécifique, $a$ fortiori dans le contexte d'une approche réellement pluridisciplinaire de cet objet. En effet, les chercheurs qui travaillaient sur la question provenaient d'espaces disciplinaires variés, depuis les Film Studies, les Media Studies, les Cultural Studies, l'histoire, la sociologie, l'anthropologie, la communication, l'économie, le management, les sciences politiques, etc. Cette particularité n'était pas propre à faciliter les échanges, dans la mesure où la recherche se structure largement, que ce soit en termes de publications ou d'événements scientifiques, autour de logiques disciplinaires. D'où l'intérêt d'un réseau qui a rapidement permis, à une échelle internationale, de fédérer des chercheurs issus 
d'horizons très divers. La constitution d'une vaste bibliographie sur le site de ce réseau (http://www.filmfestivalresearch.org/) a permis de constituer un corpus d'ouvrages et d'articles consacrés à cet objet, regroupant dans un même élan les publications produites jusqu'alors par des chercheurs souvent isolés.

Sur la base de ce premier geste, plusieurs colloques internationaux ont été organisés sur la question des festivals de films, permettant aux chercheurs virtuellement fédérés au sein de ce réseau de se rencontrer. On peut citer le rôle joué par les conférences annuelles du NECS (European Network for Cinema and Media Studies), au sein desquelles s'est constitué un groupe intitulé « Film Festival Research Workgroup » : cet affichage, doublé de la proposition régulière de panels et de Workshops sur les festivals de films, a confirmé la structuration de ce champ de recherche dans le domaine des études cinématographiques et audiovisuelles. Parallèlement, quelques conférences internationales intégralement consacrées à la question furent organisées, à l'image de la conférence « Film Festivals Cartography » organisée à Modène, en Italie, en 2014.

$\mathrm{Si}$, dorénavant, des chercheurs travaillant sur un objet commun étaient capables de s'identifier, se retrouvaient régulièrement, échangeaient sur leurs travaux, le nouveau champ disciplinaire demandait encore à être cerné. Comme pour donner corps à ce projet, deux recueils de « textes fondateurs » ont été publiés, en 2013 et 2016, tentatives que l'on peut clairement lier à la volonté de poser les bases épistémologiques de ce champ de recherche en construction (Iordanova 2013; De Valck 2016). À l'aune de ces publications, définir ce que sont les « Film Festivals Studies» demeure néanmoins une tâche complexe. Les différents textes qui tentent, individuellement de proposer une méthodologie apte à l'étude des festivals de films traduisent, mis bout à bout, une diversité des approches, des disciplines convoquées, des modèles théoriques engagés, sans que n'émerge en fin de parcours une quelconque unité. Aussi, s'il fallait définir aujourd'hui ce que sont réellement les Film Festivals Studies il faudrait chercher conformément au projet des Cultural Studies - au croisement de ces approches très diverses, lesquelles traversent, chacune selon leurs objectifs spécifiques, ce même objet.

Avant les années 1980, les articles qui abordent la question des festivals de film sont rédigés par des journalistes, lesquels abordent avant tout la question au gré d'une approche critique et esthétique des films présentés. Le large corpus de textes né de cette 
production représente aujourd'hui une source d'un grand intérêt pour qui s'intéresse à l'histoire des festivals, même si rares sont ceux qui tentent une réflexion sur le phénomène festivalier lui-même.

Parmi les textes fondateurs du champ, au cours de cette période primitive, on peut évoquer la publication en 1961 du célèbre texte d'André Bazin (1961), «Du festival considéré comme un ordre », où il décrit avec humour le statut du critique baigné dans le monde du festival de Cannes. Bazin amorce ici une analyse sociologique du statut du festivalier, plongé dans un " espace hors du monde et hors du temps », que prolongeront ensuite d'autres travaux. Cette perspective reste cependant isolée à l'époque. Les premières recherches universitaires qui abordent la question festivalière sont le plus souvent consacrées à une manifestation isolée, entremêlant un point de vue historisant et une observation participante, complétée par des interviews auprès de l'équipe organisatrice. Si tous les grands festivals internationaux vont être l'objet de travaux de ce type (Jacobsen 1990 ; Bart 1997 ; Smith 2001 ; Roddolo 2003, etc.), nombreuses sont, en France comme à l'étranger, les publications qui s'intéressent au Festival de Cannes, en tant que principal acteur du secteur à l'international.

Certains chercheurs, dans leur exploration de ces grandes manifestations internationales, vont s'intéresser plus particulièrement à la dimension géostratégique de leur activité, à la façon dont se structurent, autour des festivals, les relations entre les différents pays qui y sont - ou pas - représentés. Une des recherches les plus intéressantes dans cette perspective est celle de Loredana Latil (2013) sur le Festival de Cannes, dans son livre issu de sa thèse d'histoire, où elle montre comment l'histoire de cette manifestation ne peut être séparée d'une histoire strictement diplomatique, faite de compromis - voire de compromissions -, d'ajustements constants en fonction de réguliers rééquilibrages géopolitiques. La thèse de doctorat de Stefano Pisù, publiée en 2013, adopte de nouveau cette perspective, analysant les relations entretenues par la Biennale de Venise avec l'URSS. Le chercheur italien a depuis élargi ce regard à d'autres manifestations (Berlin, Cannes, Karlovy Vary, Moscou...) pour essayer de comprendre comment l'histoire internationale contemporaine pouvait être appréhendée au prisme des grands festivals internationaux (Pisù 2016). 
Beaucoup d'autres travaux de qualité ont été réalisés dans cette perspective mêlant histoire et géopolitique dont, en France, ceux de Caroline Moine. Cette perspective particulière traverse en outre la question des identités nationales, la façon dont elles peuvent être travaillées par les festivals. À titre d'exemple, Liz Czach (2004) a fondé sa recherche sur cette question consistant à savoir comment les festivals de films pouvaient être considérés comme l'un des mécanismes institutionnels destinés à fonder un « cinéma national ». Elle étudie spécifiquement le cas canadien, analysant la façon dont le Toronto Film Festival contribue à fédérer un sentiment de l'existence d'un " cinéma canadien ", dans le contexte d'un sentiment nationaliste renforcé au cours des années 1970. Ces travaux vont trouver une continuité dans ceux de Diane Burgess, dont la thèse, datée de 2008, se penche de nouveaux sur les festivals canadiens pour comprendre comment, articulant des intérêts économiques du marché international, des dimensions de diplomatie culturelle et des aspects cinéphiliques, ils ambitionnent une programmation autonome, liée au marché du cinéma indépendant, tout en cherchant à prendre leurs distances par rapport à d'Hollywood. L'idée ici développée d'une autonomie, à la fois identitaire, artistique, économique et culturelle, face à Hollywood, offre une clé de compréhension extrêmement intéressante du phénomène festivalier et de la constitution d'un ordre international.

Nombreux sont parallèlement les auteurs qui travaillent sur la notion de « légitimation » des œuvres, donc sur le capital symbolique dont bénéficient d'une part les festivals, en tant que dispositif, et les films en tant qu'œuvres distinguées, souvent dans une perspective bourdieusienne. Certains auteurs cherchent, dans la continuité de cette perspective, à caractériser le cadre de réception spécifique à l'univers festivalier, ainsi que les rites sociaux propres à cet univers singulier. Dans le champ des Film Studies, Bill Nichols a publié dès 1994 des articles qui montrent comment les nouvelles formes cinématographiques sont perçues, interprétées, dans le cadre spécifique de l'expérience festivalière :

Le cadre festivalier induit un sentiment de déférence, mais aussi d'humilité, de curiosité, de réceptivité, récompensé par la « découverte » d'un système, un ensemble de sens codés rendus intelligibles. L'accumulation de détails, le recoupement entre les personnes (acteurs), les lieux et les choses, les analogies entre les thèmes, les points communs entre les structures, les préoccupations, les rythmes, les emphases et 
omissions contribuent à une superposition progressive des textes, dont de nouvelles significations émergent à la surface. [pp. 80-81]

Dans ce travail, Bill Nichols prend en considération la spécificité du dispositif en luimême : il développe l'idée selon laquelle, dans une perspective que l'on pourrait qualifier de sémiopragmatique, le dispositif festivalier modifie les conditions de réception des produits cinématographiques. Cette approche trouve des prolongements dans les travaux de Daniel Dayan (2000), affilié au CNRS et à l'EHESS, qui emprunte, quant à lui, le concept de « cadre de l'expérience » à Erwing Goffman pour analyser le processus social à l'œuvre dans les festivals de films. Adoptant une démarche ethnographique, il étudie le cadre festivalier offert par le festival de Sundance. Il caractérise la dimension « d'expérience collective » [behavioural sequences] propre à la fréquentation festivalière, analysant les règles qui dictent dans ce contexte les comportements : pour lui, c'est la ritualisation $\mathrm{du}$ comportement $\mathrm{du}$ festivalier qui participe d'une légitimation de la manifestation et des œuvres qu'elle expose.

Élargissant cette perspective sociologique, Emmanuel Ethis, dans l'ouvrage qu'il a dirigé en 2001 sous le titre Aux marche du palais. Le festival de Cannes sous le regard des sciences sociales, développe une réflexion relative au dispositif festivalier appréhendé de façon globale, à la façon dont l'événement - envisagé dans l'espace et dans le temps - ne peut être compris que dans ses interactions avec le projet tel qu'énoncé par les organisateurs, mais aussi avec le monde du cinéma dans son ensemble, dans ses aspects économiques, culturels, médiatiques. Il montre aussi comment cet univers singulier répond à ses propres rituels (la montée des marches...), ses propres règles (le système des accréditations...), etc. La typologie qu'il propose au sein des festivaliers (les professionnels, les cinéphiles « accrédités » et ceux qui ne le sont pas...) participe de la construction théorique de cette micro-société, régie par ses règles propres.

Cette mise en avant du dispositif dans la compréhension du phénomène festivalier amène un certain nombre d'auteurs à considérer les festivals comme une "institution ». Dans un travail universitaire mené en 2003, Julian Stringer précise que pour lui, «le festival de films, aujourd'hui, constitue l'une des institutions-clé par lesquelles le monde contemporain du cinéma circule et peut être compris » [p. 60], d'où l'importance d'en 
étudier la logique organisationnelle, selon une perspective institutionnelle. Cette perspective a ouvert la voie à des travaux dans le domaine du management, interrogeant la façon dont l'approche organisationnelle pouvait alimenter une réflexion sur les festivals de films, à l'image des publications de Charles-Clemens Ruling et Jesper Strandgaard Pedersen (2010). Cette approche se fonde sur divers constats, parmi lesquels le fait qu'organiser un festival suppose l'intervention et l'interaction d'acteurs très différents (réalisateurs, producteurs, journalistes, financiers, juristes, distributeurs, représentants de l'économie touristique, organisateurs du festival, etc.). Les théories organisationnelles développent ainsi un certain nombre de pistes propres à appréhender la nature des relations qui se nouent entre ces individus. La question des ressources humaines traverse logiquement ces réflexions, les festivals recourant à des employés de nature très diverse (salariés, stagiaires, bénévoles, intérimaires, etc.). La perspective organisationnelle amène aussi à s'interroger sur l'identité que se forge l'organisationfestival, par ses stratégies de programmation, mais aussi, pour les plus grands d'entre eux, ses stratégies d'accréditation auprès de la FIAPF - Fédération internationale des associations des producteurs de films (Moine 2013).

L'étude des festivals de films ne pouvait, enfin, faire l'impasse sur la perspective économique, afin de comprendre le rôle que jouent les festivals au niveau de l'économie locale, mais aussi - ce qui est plus spécifique à la question strictement cinématographique - dans la chaîne de valeur de l'économie du film. Rares sont les études menées par des économistes sur ces questions spécifiques, hormis pour étudier le rôle joué par les marchés du film (Kerin, Cron 1987 ; Moeran, Strandgaard 2012).

Pour conclure ce rapide tour d'horizon des différents types de travaux entrepris, on pourrait évoquer une dernière perspective consistant à appréhender le monde festivalier en tant que réseau international. Cette idée d'un «festival network» a en particulier marqué les travaux de deux chercheurs de l'université d'Amsterdam, Thomas Elsaesser en 2005, de Marijke De Valck en 2007. Tous deux ont travaillé à développer un modèle théorique du circuit international des festivals de films, sur la base de réflexions qui avaient commencé à se développer dans les années 1990 autour de chercheurs comme Bill Nichols, et qui avançaient l'idée que les festivals de film ne peuvent être appréhendés dans la spécificité de leur nationalité, et qu'au contraire il fallait les 
comprendre dans un système global. Marijke De Valck envisage les festivals comme autant de "points de connexion » au sein d'un circuit cinématographique européen fonctionnant « contre », mais aussi avec Hollywood, et ce dans des perspectives à la fois géopolitiques, commerciales et culturelles : c'est, pour elle, au cœur de la vie festivalière que se tissent les liens politiques entre nations à propos du cinéma, que se développent les stratégies économiques, mais aussi que sont testées les nouvelles pratiques cinéphiles. Cet ouvrage, du fait aussi du rôle moteur joué par Marijke De Valck dans la fédération des Film Festival Studies, a eu un impact important dans les recherches au cours des années suivantes, posant en quelque sorte les prémisses d'une structuration de la discipline autour d'une perspective commune.

On peut cependant interroger les fondements inhérents à cette approche, consistant à limiter le périmètre festivalier aux grands festivals internationaux et à rejeter la notion de «nationalité » appliquée au cinéma, qui tend à limiter un certain nombre de recherches dans une vision finalement restrictive face à l'ampleur du phénomène. Plusieurs chercheurs, à l'image de Dina Iordanova, défendent l'idée selon laquelle les festivals de film ne peuvent être réfléchis qu'au gré d'une perspective transnationale. Or, comme on l'a largement montré sur la base du cas français dans la première partie de ce texte, ce déni de l'ancrage national de la culture festivalière (le système législatif, étatique, politique dans lequel ils se déploient, l'histoire des médiations cinéphiles dans laquelle ils s'inscrivent, l'environnement industriel et commercial qui y prévaut, etc.) peut apparaitre comme un frein à la pleine compréhension du phénomène. L'autre élément qui pose un problème de fond est lié à cette focalisation qu'opèrent nombre de chercheurs sur les grands festivals internationaux, ceux en particulier qui sont homologués par la FIAPF : les fonctions de marché international du film que jouent effectivement ces grandes institutions sont alors survalorisées dans les recherches, au détriment des fonctions de laboratoire cinéphilique, de création de lien social, de dynamisation des territoires qu'assument les très nombreuses autres manifestations, à la portée souvent plus locale ou régionale, mais qui enrichissent considérablement le concept même de «festival du film ». Suivant la même démarche, on pourra regretter que l'étude récurrente des festivals appartenant au circuit de la FIAPF focalise le propos sur l'Europe occidentale et l'Amérique du Nord, au détriment des autres régions du monde où, pourtant, le secteur 
festivalier est parfois animé d'un dynamisme tout particulier, heureusement mis en lumière par quelques chercheurs contemporains.

Bilan : les défis des Film Festival Studies aujourd'hui

Comme le souligne ce très bref aperçu transversal des travaux qui ont marqué la construction de ce champ de recherche, la compréhension du phénomène festivalier implique le recours à des approches disciplinaires très variées. S'il est difficile aujourd'hui d'affirmer que les Film Festival Studies ont pris leur envol, c'est justement parce que la synthèse entre ces approches ne nous semble pas avoir été effectuée. La construction du champ de recherche n'a été pensée jusqu'alors que comme bout à bout de travaux souvent éclectiques, qui ne se répondent que ponctuellement. Le passage d'une communauté de chercheurs rassemblés au sein d'un même intitulé au gré d'une affirmation pluridisciplinaire telle qu'elle prévaut aujourd'hui à la mise en œuvre d'une véritable interdisciplinarité, voire d'une transdisciplinarité, dans les approches conjuguées offrirait probablement un cadre de développement plus propice à la pleine affirmation de ce champ de recherche. La création d'un laboratoire de recherche, ou d'un observatoire international, pleinement investi dans ces réflexions constituerait probablement un levier intéressant dans ce cadre, un préalable, peut-être, au passage des Film Festival Studies de l'enfance à l'âge adulte.

Christel Taillibert Université Côte d'Azur

\section{Références}

Bart, P. (1997) Cannes, Fifty Years of Sun, Sex \& Celluloid: Behind the Scenes at the World's Most Famous Film Festival. New York : Hyperion.

Bazin, A. (1961) «Du festival considéré comme un ordre », in Qu'est-ce que le cinéma? T. 3, Cinéma et sociologie. Paris : Édition du Cerf, (posth.).

Burgess, D. (2008) Negotiating value: a Canadian perspective on the international film festival. Thèse de doctorat, University of British Columbia.

CNC (2016) La production cinématographique et audiovisuelle en région. Paris : CNC, Les études du CNC.

Czach, L. (2004) « Film Festivals, Programming and the Building of a National Cinema », The Moving Image, 4/1 : 76-88. 
Dayan, D. (2000) «Looking for Sundance : The Social Construction of a Film Festival», in Bondebjerg, Ib (ed.), Moving Images, Culture and the Mind, 43-52. Londres : University of Luton Press.

De Baecque, A. (2003) La cinéphilie - Invention d'un regard, histoire d'une culture, 19441968. Paris : Fayard.

De Valck, M., Hagener, M. (ed.) (2005) Cinephilia : Movies, Love and Memory. Amsterdam : Amsterdam University Press.

De Valck. M. (2007) Film Festivals : From European Geopolitics to Global Cinephilia. Amsterdam : Amsterdam University Press.

De Valck, M., Brendan K., Loist, S. (eds) (2016). Film Festivals, History, Theory, Method, Practice. Londres : Routledge.

Elsaesser, T. (2005) European Cinema : Face to Face with Hollywood. Amsterdam : AUP.

Ethis, E. (ed.) (2001) Aux marches du palais, Le festival de Cannes sous le regard des sciences sociales. Paris : la Documentation française.

Ethis E. (2007) «Le cinéma, cet art subtil du rendez-vous », Communication et langages, L'énonciation éditoriale en question, $154: 11-21$.

Flichy, P. (2010) Le sacre de l'amateur : sociologie des passions ordinaires à l'ère numérique. Paris : Seuil.

Getz, D. (2010) «The Nature and Scope of Festival Studies », International Journal of Event Management Research, 5/1 http://ijemr.org/wp-content/uploads/2014/10/Getz.pdf

Gumplowicz, P. (1987) Les travaux d'Orphée. 150 ans de vie musicale amateur en France. Harmonies, chorales, fanfares. Paris : Aubier.

Iordanova, D. (2013) The Film Festival Reader, St Andrews : St Andrews Film Studies.

Jacobsen, W. (1990) Berlinale: Berlin International Film Festival. Berlin : Argon.

Kerin R. A. ; Cron W. L. (1987) «Assessing Trade Show Functions and Performance: An Exploratory Study », Journal of Marketing 51/3: 87-94.

Latil, L. (2005) Le festival de Cannes sur la scène internationale. Paris : Nouveau Monde.

Moine, C. (2013) «La Fédération internationale des associations de producteurs de films : un acteur controversé de la promotion du cinéma après 1945 », Le Mouvement Social 243 : 91-103.

Moeran, B., Strandgaard Pedersen, J. (2012) Negotiating Values in the Creative Industries : Fairs, Festivals and Competitive Events. Cambridge : Cambridge University Press.

Nichols, B. (1994) " Global Image Consumption in the Age of Late Capitalism », East-West Journal, VIII/1 : 68-85.

Pisù, S. (2013) Stalin a Venezia: l'URSS alla Mostra del cinema fra diplomazia culturale et scontro ideologico (1932-1953). Soveria Mennelli : Rubbettino.

Pisù, S. (2016) Il XX secolo sul red carpet. Politica, economia e cultura nei festival internazionali del cinema (1932-1976). Milano : Franco Angeli.

Roddolo E. (2003) La Biennale: Arte, Scandali e Storie in Laguna. Venezia : Marsilio.

Rüling, C.-C. ; Strandgaard Pedersen, J. (2010) «Film festival research from an organizational studies perspective », Scandinavian Journal of Management, $26: 318-323$.

Smith, L. (2001) Party in a Box: The story of the Sundance Film festival. Sal Lake City : Gibbs-Smith Publisher.

Stringer, J. (2003) « Regarding Film Festivals : Introduction » Reproduit dans : Iordanova, D. (ed.) (2003) The Film Festival Reader, pp. 59-68. St Andrews : St Andrews Film Studies.

Taillibert, C. (2009) Tribulations festivalières - Les festivals de cinéma et audiovisuel en France. Paris : L'Harmattan. 